\title{
Numerical evaluation of one-loop QCD amplitudes
}

\author{
Simon Badger* \\ Niels Bohr International Academy and Discovery Center, The Niels Bohr Institute, \\ University of Copenhagen, Blegdamsvej 17, DK-2100 Copenhagen, Denmark \\ E-mail: badger@nbi.dk

\section{Benedikt Biedermann} \\ Humboldt-Universität zu Berlin, Institut für Physik, \\ Newtonstraße 15, D-12489 Berlin, Germany \\ E-mail: benedikt.biedermann@physik.hu-belin.de
}

\section{Peter Uwer}

Humboldt-Universität zu Berlin, Institut für Physik, Newtonstraße 15, D-12489 Berlin, Germany

E-mail: peter.uwer@physik.hu-belin.de

\section{Valery Yundin}

Niels Bohr International Academy and Discovery Center, The Niels Bohr Institute, University of Copenhagen, Blegdamsvej 17, DK-2100 Copenhagen, Denmark

E-mail: yundin@nbi.dk

We present the implementation of the numerical generalized unitarity algorithm in the public code NJET. As phenomenological applications we discuss three and four jet production at the LHC to next-to-leading order accuracy in massless QCD.

Loops and Legs in Quantum Field Theory - 11th DESY Workshop on Elementary Particle Physics, April 15-20, 2012

Wernigerode, Germany

\footnotetext{
* Speaker.
} 


\section{Introduction}

Particle production at hadron colliders is dominated by large amounts of QCD radiation resulting in large backgrounds to new physics searches. When calculating such processes using perturbative QCD corrections beyond leading order can be large and next-to-leading order is required to obtain quantitative predictions. Until recently evaluation of the required virtual corrections were a major bottleneck preventing a systematic extension of existing studies to NLO accuracy.

Over the last decade methods for the computation of multi-particle one-loop amplitudes have seen considerable improvement. New techniques like unitarity [1, 2], generalized unitarity [3], integrand reduction [4] and recursive tensor reduction [5, 6] have alleviated a long standing bottleneck in NLO computations. The rapid growth in the size of analytic expressions can be avoided by performing algebraic steps numerically. At this time several different approaches have been applied successfully to $2 \rightarrow 4$ processes ${ }^{1}$ and to a handful of cases with even higher multiplicities [8-11]. A number of groups have made substantial progress towards the complete automation of NLO corrections [12 - 17] of which some elements are available in public codes [16-20].

Precision predictions for di-jet production at Hadron colliders have been known for twenty years [21, 22]. The full three jet computation was only completed in 2003 [23], though the pure gluonic contribution was known previously [24]. Full predictions for four jet production are complicated by the large amounts of gluonic radiation which cause theoretical computations to become extremely difficult. Using the benefits of an on-shell approach the BLACKHAT collaboration were recently able to make the first predictions [25]. Some studies using parton shower matching [26, 27] have recently been carried out for inclusive jet and di-jet productions [28, 29].

In these proceedings we present the recently released C++ library NJET [17] and the application to three and four jet production at the LHC with a centre of mass energy of $\sqrt{s}=8 \mathrm{TeV}$ [30]. After highlighting some key features of the program we discuss some differential distributions of multi-jet production at the LHC at next-to-leading order produced by combining NJET with the SHERPA Monte-Carlo event generator [31].

\section{One-loop multi-parton amplitudes}

We take a generalized unitarity approach to the construction of one-loop amplitudes in massless QCD where the fundamental building blocks are gauge invariant ordered amplitudes known as primitive amplitudes. These primitive amplitudes are first expanded in a basis of known scalar integral functions with up to four external legs,

$$
\begin{aligned}
& A_{n}^{(1)}\left(p_{1}, \cdots, p_{n}\right)=\sum_{i_{1}<i_{2}<i_{3}<i_{4}} C_{4 ; i_{1} i_{2} i_{3} i_{4}} I_{4 ; i_{1} i_{2} i_{3} i_{4}}+\sum_{i_{1}<i_{2}<i_{3}} C_{3 ; i_{1} i_{2} i_{3}} I_{3 ; i_{1} i_{2} i_{3}} \\
& \quad+\sum_{i_{1}<i_{2}-1} C_{2 ; i_{1} i_{2}} I_{2 ; i_{1} i_{2}}+\sum_{i_{1}<i_{2}<i_{3}<i_{4}} C_{4 ; i_{1} i_{2} i_{3} i_{4}}^{[4]}\left(-\frac{1}{6}\right) \\
& \quad+\sum_{i_{1}<i_{2}<i_{3}} C_{3 ; i_{1} i_{2} i_{3}}^{[4]}\left(-\frac{1}{2}\right)+\sum_{i_{1}<i_{2}-1} C_{2 ; i_{1} i_{2}}^{[4]} I_{2 ; i_{1} i_{2}}\left(-\frac{\left(p_{i_{1}}+\cdots+p_{i_{2}-1}\right)^{2}}{6}\right)+\mathscr{O}(\varepsilon),
\end{aligned}
$$

\footnotetext{
${ }^{1}$ The latest Les Houches SM and NLO multi-leg Working group report [7] contains a good overview of the growing number of phenomenological studies.
} 
where $I_{p ; X}$ are the integral functions and $C_{p ; X}$ are process dependent rational coefficients.

The numerical generalized unitarity algorithm [12, 32-35] implemented in NJET is based on the NGLUON library for multi-gluon primitive amplitudes [16]. The scalar integrals are obtained via the QCDLOOP/FF library [36, 37] and quadruple precision for rescuing unstable phase space points is implemented via the QD library [38]. The updated version includes an implementation of arbitrary multiplicity multi-fermion primitive amplitudes and the full colour summation for all partonic sub-processes for up to five jet production. An interface via the Binoth Les Houches Accord [39] allows the library to be linked directly to external Monte-Carlo programs.

To compute full colour summed virtual corrections it is necessary to match the primitive amplitudes to the $S U\left(N_{c}\right)$ colour structures appearing in the full amplitude. We apply an algorithm which uses matching of the ordered primitives to Feynman diagrams to achieve this goal along the lines of the procedures employed in Refs. [40, 41]. The total number of primitives contributing to the coloured amplitude is significantly reduced by taking "Furry's theorem" into account for the fermion loops. Further details can be found in Ref. [17].

The speed and accuracy of any numerical approach are clearly very important. Inside NJET we use a momentum scaling check to estimate numerical instabilities and switch to quadruple precision in case a user specified number of valid digits is not achieved. By using super-symmetric identities, a cache system for helicity and permutation sums and de-symmetrized colour sums in the complicated gluonic channels average times of the order of 2 seconds per event were achieved during the phase-space integration of $p p \rightarrow 4$ jets.

\section{Multi-jet production at the LHC with NJET and SHERPA}

Predictions for multi-jet observables at NLO have recently been made by combining the NJET library with the SHERPA Monte-Carlo event generator. In this study the earlier predictions by the BLACKHAT collaboration for the LHC at $\sqrt{s}=7 \mathrm{TeV}$ [25] were verified and new predictions for the LHC at $\sqrt{s}=8 \mathrm{TeV}$ were obtained, including differential distributions in transverse momentum, $p_{T}$, and rapidity. At NLO the differential cross section receives contributions from both unresolved real radiation, $d \sigma_{n+1}^{\text {real }}$, and the virtual loop corrections, $d \sigma_{n}^{\text {virtual }}$,

$$
d \sigma^{\mathrm{NLO}}=d \sigma_{n}^{\mathrm{born}}+d \sigma_{n}^{\text {virtual }}+d \sigma_{n}^{\hat{\mathrm{S}}}+d \sigma_{n+1}^{\mathrm{real}}-d \sigma_{n+1}^{\mathrm{S}}+d \sigma_{n}^{\mathrm{F}}
$$

The cancellation of infra-red singularities was performed using Catani-Seymour dipole subtraction [42] which determines the form of the subtraction $\left(d \sigma_{n+1}^{\mathrm{S}}\right)$, integrated subtraction $\left(d \sigma^{\hat{\mathrm{S}}_{n}}\right)$, and factorization $\left(d \sigma_{n}^{\mathrm{F}}\right)$ terms. This procedure is implemented into SHERPA [43]. The real and born matrix elements were obtained using AMEgIC++ [44] and Comix [45]. The anti-kt jet clustering algorithm with $R=0.4$ was used via the FASTJET library [46, 47]. The MSTW2008 PDF set was used [48] in the fixed five flavour scheme. All effects from top quarks have been neglected though they are expected to be small. Asymmetric $p_{T}$ cuts were applied with $p_{T}>80 \mathrm{GeV}$ for the leading jet and $p_{T}>60 \mathrm{GeV}$ for all subsequent jets. Rapidity cuts of $|\eta|<2.8$ were also taken. The renormalization and factorization scales were taken to be equal and proportional the sum of the transverse momenta of the final state partons, $\hat{H}_{T}$. This dynamical scale was chosen with a central value of $\mu_{R}=\hat{H}_{T} / 2$ and scale variations estimated using $\mu_{R}=\hat{H}_{T} / 4$ and $\mu_{R}=\hat{H}_{T}$. 
At $\sqrt{s}=8 \mathrm{TeV}$ the total cross sections for three and four jet production are found to be,

$$
\begin{array}{llrl}
\sigma_{3}^{8 \mathrm{TeV}-\mathrm{LO}}=126.65(0.05)_{-40.40}^{+66.56} \mathrm{nb}, & & \sigma_{3}^{8 \mathrm{TeV}-\mathrm{NLO}}=72.57(0.16)_{-28.08}^{+2.71} \mathrm{nb}, \\
\sigma_{4}^{8 \mathrm{TeV}-\mathrm{LO}}=14.36(0.01)_{-5.6}^{+10.38} \mathrm{nb}, & \sigma_{4}^{8 \mathrm{TeV}-\mathrm{NLO}}=8.15(0.09)_{-3.24}^{+0.0} \mathrm{nb} .
\end{array}
$$

The $p_{T}$ and rapidity distributions for the fourth leading jet (ordered by $p_{T}$ ) are shown in Fig. 1. We can clearly observe the average size of the NLO corrections is large at the order of $50 \%$ and the scale variations are significantly reduced.
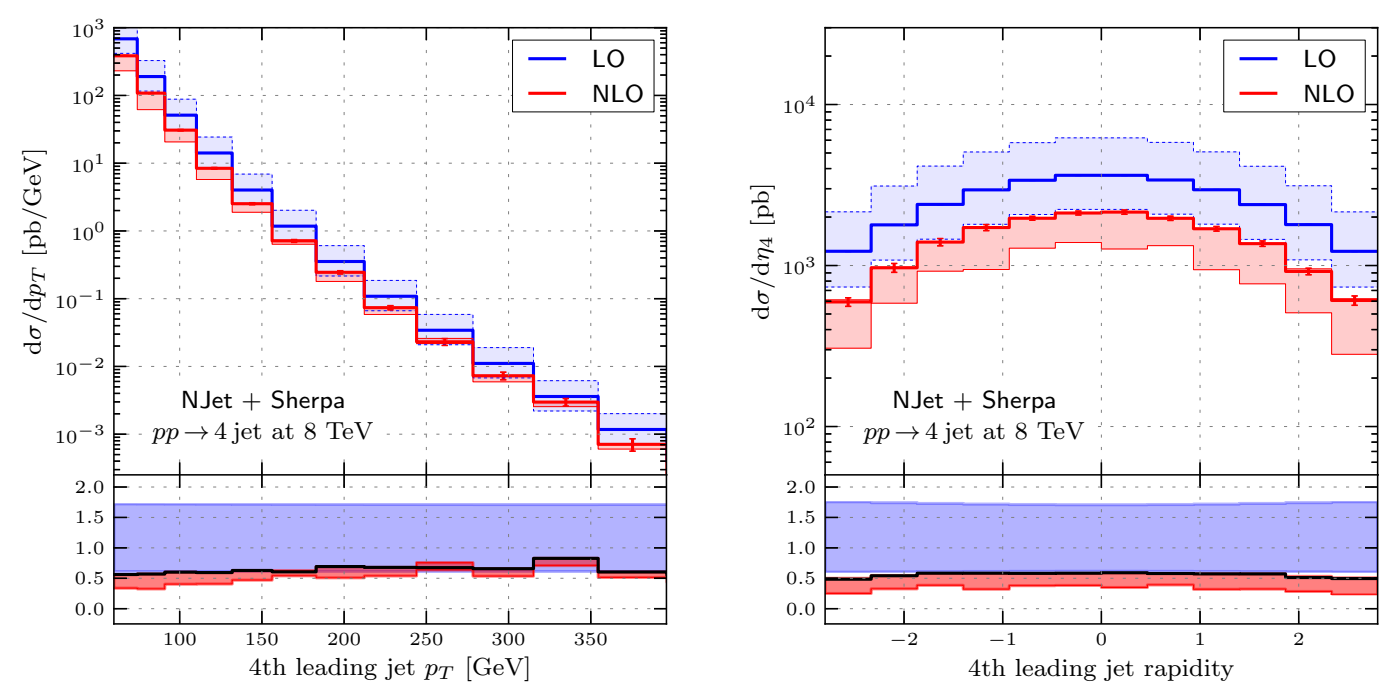

Figure 1: $p_{T}$ and rapidity distributions for the $4^{\text {th }}$ leading jet in $p p \rightarrow 4$ jets. LO predictions are shown in blue with the shaded blue band indicating scale variations. NLO predictions are shown red. The lower plot shows the ratio of NLO/LO.

\section{Outlook}

We have presented results for multi-jet production at hadron colliders computed at NLO using the NJET C++ library and the SHERPA Monte-Carlo program. The on-shell generalized unitarity algorithm is well suited for such processes due to the large amount of gluonic radiation which is difficult to deal with when using alternative methods. For the LHC running with a centre-of-mass energy of $\sqrt{s}=8 \mathrm{TeV}$ we observe sizeable corrections at NLO. As discussed in Ref. [30] a large proportion of these corrections can be attributed to the NLO value of $\alpha_{s}$ coming from the parton distribution functions. In low $p_{T}$ regions the fixed order perturbative series is known to break down because of large logarithms coming from soft gluons. Future studies using parton shower matching would be an interesting way to estimate the size of these effects.

\section{References}

[1] Z. Bern, L. J. Dixon, D. C. Dunbar, and D. A. Kosower Nucl.Phys. B425 (1994) 217-260, [hep-ph/9403226].

[2] Z. Bern, L. J. Dixon, D. C. Dunbar, and D. A. Kosower Nucl.Phys. B435 (1995) 59-101, [hep-ph/9409265]. 
[3] R. Britto, F. Cachazo, and B. Feng Nucl.Phys. B725 (2005) 275-305, [hep-th/ 0412103 ].

[4] G. Ossola, C. G. Papadopoulos, and R. Pittau Nucl.Phys. B763 (2007) 147-169, [hep-ph/0609007].

[5] A. Denner and S. Dittmaier Nucl.Phys. B734 (2006) 62-115, [hep-ph/ 0509141$].$

[6] T. Diakonidis, J. Fleischer, J. Gluza, K. Kajda, T. Riemann, et al. Phys.Rev. D80 (2009) 036003, [0812.2134].

[7] SM AND NLO MULTILEG and SM MC Working Groups Collaboration, J. Alcaraz Maestre et al. 1203.6803 .

[8] R. Frederix, S. Frixione, K. Melnikov, and G. Zanderighi JHEP 1011 (2010) 050, [1 008.5313 ].

[9] C. Berger, Z. Bern, L. J. Dixon, F. Febres Cordero, D. Forde, et al. Phys.Rev.Lett. 106 (2011) 092001, [1009.2338].

[10] H. Ita, Z. Bern, L. Dixon, F. Febres Cordero, D. Kosower, et al. Phys.Rev. D85 (2012) 031501, [1108.2229].

[11] S. Becker, D. Goetz, C. Reuschle, C. Schwan, and S. Weinzierl Phys.Rev.Lett. 108 (2012) 032005, [1111.1733].

[12] C. Berger, Z. Bern, L. Dixon, F. Febres Cordero, D. Forde, et al. Phys.Rev. D78 (2008) 036003, [0803.4180].

[13] W. Giele and G. Zanderighi JHEP 0806 (2008) 038, [0 805 . 2152].

[14] V. Hirschi, R. Frederix, S. Frixione, M. V. Garzelli, F. Maltoni, et al. JHEP 1105 (2011) 044, [1103.0621].

[15] F. Cascioli, P. Maierhofer, and S. Pozzorini Phys.Rev.Lett. 108 (2012) 111601, [1111. 5206 ].

[16] S. Badger, B. Biedermann, and P. Uwer Comput.Phys.Commun. 182 (2011) 1674-1692, [1011.2900].

[17] S. Badger, B. Biedermann, P. Uwer, and V. Yundin 1209.0100.

[18] P. Mastrolia, G. Ossola, T. Reiter, and F. Tramontano JHEP 1008 (2010) 080, [1 006.0710 ].

[19] G. Bevilacqua, M. Czakon, M. Garzelli, A. van Hameren, A. Kardos, et al. 1110.1499.

[20] G. Cullen, N. Greiner, G. Heinrich, G. Luisoni, P. Mastrolia, et al. Eur.Phys.J. C72 (2012) 1889, [1111.2034].

[21] S. D. Ellis, Z. Kunszt, and D. E. Soper Phys.Rev.Lett. 69 (1992) 1496-1499.

[22] W. Giele, E. N. Glover, and D. A. Kosower Nucl.Phys. B403 (1993) 633-670, [hep-ph/9302225].

[23] Z. Nagy Phys.Rev.Lett. 88 (2002) 122003, [hep-ph/0110315].

[24] W. B. Kilgore and W. Giele Phys.Rev. D55 (1997) 7183-7190, [hep-ph/9610 433].

[25] Z. Bern, G. Diana, L. Dixon, F. Febres Cordero, S. Hoeche, et al. 1112 . 3940.

[26] S. Frixione and B. R. Webber JHEP 0206 (2002) 029, [hep-ph/ 0204244$].$

[27] P. Nason JHEP 0411 (2004) 040, [hep-ph / 0409146 ].

[28] S. Alioli, K. Hamilton, P. Nason, C. Oleari, and E. Re JHEP 1104 (2011) 081, [1 012 . 3380 ].

[29] S. Hoeche and M. Schonherr 1208.2815. 
[30] S. Badger, B. Biedermann, P. Uwer, and V. Yundin 1209.0098.

[31] T. Gleisberg, S. Hoeche, F. Krauss, M. Schonherr, S. Schumann, et al. JHEP 0902 (2009) 007, [0811.4622].

[32] R. Ellis, W. Giele, and Z. Kunszt JHEP 0803 (2008) 003, [0 708 . 2398].

[33] W. T. Giele, Z. Kunszt, and K. Melnikov JHEP 0804 (2008) 049, [0 801 . 22 37].

[34] D. Forde Phys.Rev. D75 (2007) 125019, [0 704 . 1835].

[35] S. Badger JHEP 0901 (2009) 049, [0 806 . 4600].

[36] G. van Oldenborgh Comput.Phys.Commun. 66 (1991) 1-15.

[37] R. K. Ellis and G. Zanderighi JHEP 0802 (2008) 002, [0 712 . 1851 ].

[38] Y. Hida, X. S. Li, and D. H. Bailey. http://crd.lbl.gov/ dhbailey/mpdist/, 2010.

[39] T. Binoth, F. Boudjema, G. Dissertori, A. Lazopoulos, A. Denner, et al. Comput.Phys.Commun. 181 (2010) 1612-1622, [1001.1307]. Dedicated to the memory of, and in tribute to, Thomas Binoth, who led the effort to develop this proposal for Les Houches 2009.

[40] R. Ellis, Z. Kunszt, K. Melnikov, and G. Zanderighi 1105.4319.

[41] H. Ita and K. Ozeren JHEP 1202 (2012) 118, [1111.4193].

[42] S. Catani and M. Seymour Phys.Lett. B378 (1996) 287-301, [hep-ph/9602277].

[43] T. Gleisberg and F. Krauss Eur.Phys.J. C53 (2008) 501-523, [0 709.2881$].$

[44] F. Krauss, R. Kuhn, and G. Soff JHEP 0202 (2002) 044, [hep-ph/ 0109036 ].

[45] T. Gleisberg and S. Hoeche JHEP 0812 (2008) 039, [0 808 . 3674 ].

[46] M. Cacciari, G. P. Salam, and G. Soyez JHEP 0804 (2008) 063, [0 802 . 1189 ].

[47] M. Cacciari, G. P. Salam, and G. Soyez Eur.Phys.J. C72 (2012) 1896, [1111. 6097 ].

[48] A. Martin, W. Stirling, R. Thorne, and G. Watt Eur.Phys.J. C63 (2009) 189-285, [0 901.0002 ]. 